\title{
Téoros
}

Revue de recherche en tourisme

\section{Québec, capitale de la neige}

\section{Jean Lapointe}

Volume 12, numéro 1, mars 1993

Québec, capitale et région touristiques

URI : https://id.erudit.org/iderudit/1078199ar

DOI : https://doi.org/10.7202/1078199ar

Aller au sommaire du numéro

Éditeur(s)

Université du Québec à Montréal

ISSN

0712-8657 (imprimé)

1923-2705 (numérique)

Découvrir la revue

Citer cet article

Lapointe, J. (1993). Québec, capitale de la neige. Téoros, 12(1), 26-29.

https://doi.org/10.7202/1078199ar d'utilisation que vous pouvez consulter en ligne.

https://apropos.erudit.org/fr/usagers/politique-dutilisation/ 


\title{
Québec, capitale de la neige
}

\author{
Jean Lapointe
}

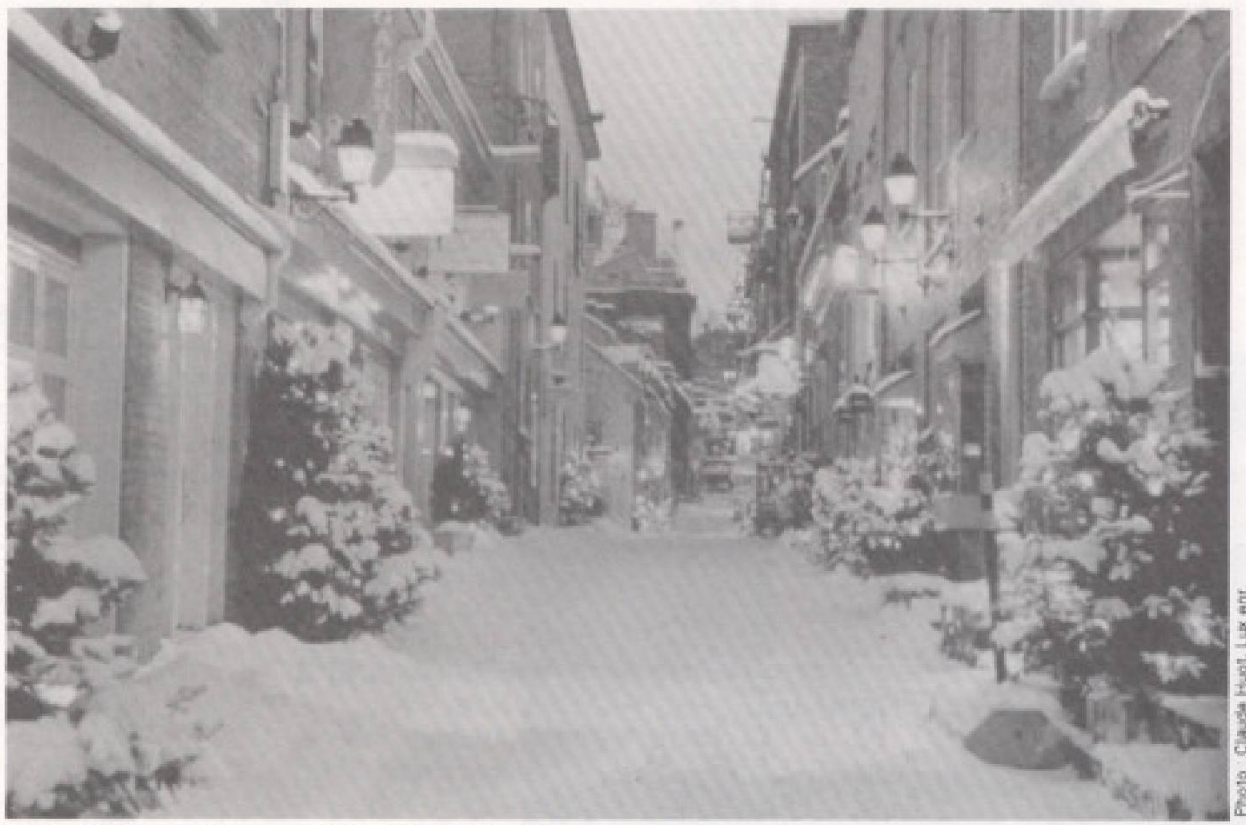

*Le Vieux-Québec sous la neige ... chame et plaisir. *

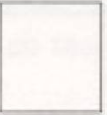

Proclamée joyau du patrimoine mondial, Québec est reconnue avant tout pour la valeur exceptionnelle de ses attraits culturelset pour son site unique en bordure du SaintLaurent. Le paysage de Québec, souvent présenté dans son décor estival, offre, pendant la période hivernale, des images et un potentiel d'exploitation touristiques dont certaines activités relèvent de l'exotisme.

En effet, à chaque hiver, une abondante couche de neige recouvre Québec et sa région qui se donnent alors des airs de fête. Les places publiques, le quartier historique et les artères commerciales se parent de sapins aux mille lumières. Le parc des champs de bataille nationaux, la Place d'Youville, la rivière Saint-Charles ainsi que la terrasse Dufferin deviennent de vastes zones récréatives où les loisirs familiaux sont à l'honneur. Le temps du Carnaval avec le palais du Bonhomme et la place de la Famille ne font qu'ajouter à la féerie déjà existante. L'ensemble de la région offre aux adeptes du sport et aux visiteurs des équipements et des activités de plein air qui surprennent autant

Monsieur Jean Lapointe est directeur de l'École de tourisme et d'hótellerie du College Mérici a Québec. par leur originalité que par leur proximité du centre-ville.

En effet, à moins de quarante minutes de la colline parlementaire, il est possible de faire du ski alpin et de randonnée dans de grandes stations touristiques ou de s'évader dans la nature en raquette ou en motoneige. La publicité du centre de ski Le Relais «à quelques centimètres de Québec $*$ est très significative à cet égard. Le parc du Mont SainteAnne, reconnu internationalement, se situe à trente minutes de Québec. Les grandes stations touristiques les pluséloignées des rives Nord et Sud se trouvent à moins d'une heure des ponts de Québec ou Pierre-Laporte.

Cette offre unique d'un produit hivernal garanti et de longue durée s'explique par un climat particulier qui règne dans la vieille capitale et ses environs. En effet, notre hiver s'échelonne en moyenne du 15 novembreau 5 avril et on y enregistre des précipitations records dans la province de Québec. A l'aéroport de Sainte-Foy, il tombe plus de $340 \mathrm{~cm}$ de neige, soit $88 \mathrm{~cm}$ de plus qu'aे Sherbrooke et $108 \mathrm{~cm}$ de plus qu'à Montréal. Lastationmét́́orologiquedeSaint-Philémon, dans les Appalaches, enregistre depuis 1982 des précipitations nivales moyennes de 470 $\mathrm{cm}$. La réserve des Laurentides qui voisine la banlieue de Québec enregistre, quant à elle. des chutes de neige estimées à $530 \mathrm{~cm}$.

Ces conditions climatiques exceptionnelles, associées au paysage particulier de Québec, ont favorisé au cours des années l'éclosion d'activités hivemales de plein air d"une telle ampleur que Québec et sa région ont mérité le titre de «Capitale de la neigew.

\section{La région de Québec}

La région de Québec s'étend sur les deux rives du fleuve Saint-Laurent. Elle est constituée delarégion métropolitaineetd'une zone périphérique couvrant un rayon de 50 $\mathrm{km}$ autour du kilomètre zéro situé sur la colline parlementaire. L'appellation grande région de Québec estde plus en plus courante et couvre l'ensemble du territoire à plus ou moins une heure de route du centre-ville ou des ponts. Cette zone naturelle englobe en grande partie les territoires des régions touristiques de Québec, de ChaudièreAppalaches et de Charlevoix.

\section{Un paysage de plaines et de monts}

La région de Québecest le point de rencontre de trois grandes zones naturelles soit les Laurentides, la plaine du Saint-Laurent et les Appalaches.

Sur la rive Nord, les premières collines des Laurentides situées à moins de quinze kilomètres du centre-ville surplombent les banlieues. En direction nord, le mont SainteAnne et le Cap Tourmente qui se jette dans le Saint-Laurent limitent trèsnettementlaplaine. La portion des Laurentides vis-à-vis Québec est une immense zone boisće, riche en coniferes et bien équipée en rivières et en lacs. Ce vaste territoire récréo-touristique est occupé en grande partie par les réserves fauniques des Laurentides et de Portneuf, du parc provincial de la Jacques-Cartier, du parc régional du Mont Sainte-Anne ainsi que par les terres qui sont des propriétés du Petit Séminaire de Québec.

La plaine du Saint-Laurent, très large et basse dans la région de Montréal, s"éleve et 
se découpe, à Québec, en plusieurs platesformes d'environ 100 mètres d'altitude de chaque côté du fleuve Saint-Laurent. C'est ainsi que les gradins des banlieues Nord, la Côtede Beaupré, l' lled'Orléans, les hauteurs de Québec et la plate-forme de la rive Sud plus développéesurplombentle fleuve SaintLaurent. La partie basse de Québec est occupée par la rivière Saint-Charles et les basses terres des côtes de Beauport et de Beaupré.

Sur la rive Sud, le relief des Appalaches se profile au loin et n'est visible de Québec que par temps clair en raison de l'étendue de la plaine. Les rivières Etchemin et Chaudières traversent un vaste paysage agricole qui contraste avec la zone des Appalaches qui renferme les sommets skiables les plus élevés du Québec (Massif du Sud - 915 m et Grande Coulee $-853 \mathrm{~m}$ ). Cet ensemble plutôt vallonneux, est reconnu pour ses traditionnelles érablières.

\section{Un climat particulier}

Généralement, les visiteurs en direction de Québec constatent avec étonnement l'augmentation progressive et marquée de la couche neigeuse qui recouvre notre région. Tôt à l'automne ou tard au printemps, les Montréalais qui empruntent les autoroutes 20 ou 40 observent la présence de neige au sol qui semble persister dans la région de Québec. Selon les données climatiques compilées entre 1951 et 1980 par Environnement Canada, Québec reçoit en moyenne annuellement $343 \mathrm{~cm}$ de neige et la durée moyenne de l'hiver y est de 141 jours, soit 19 jours de plus que la métropole.

\section{Les activités hivernales en ville}

\section{Le Carnaval de Québec}

L'activité hivernale la plus connue est sans contredit le Carnaval de Québec qui, au cours des années, a permis a la population et aux visiteurs d'apprivoiser l'hiver et de profiter de ses plaisirs. S'inspirant de la tradition des nombreux clubs de raquetteurs et de la popularité grandissante du ski alpin, le Carnaval de Québec est devenu un événement touristique majeur qui propose plus d'une centaine d'activités dont les deux grandes parades en soirće de la basse-ville et de la haute-ville.

Sous le thème La chaleur de l'hiver, la 39e édition du Camaval a eu lieu du 4 au 14 février 1993. La grande nouveauté de cette

\begin{tabular}{|lcccr|}
\hline \multicolumn{3}{c|}{$\begin{array}{c}\text { TABLEAU 1 } \\
\text { L'hiver au Québec }\end{array}$} & & \\
\hline & Début & Fin & Durée & Précipitations \\
\hline Forất Montmorency & $05 / 11$ & $15 / 04$ & $170 \mathrm{jrs}$ & $530 \mathrm{~cm}$ \\
Québec & $15 / 11$ & $05 / 04$ & $141 \mathrm{jrs}$ & $343 \mathrm{~cm}$ \\
Sherbrooke & $17 / 11$ & $28 / 03$ & $130 \mathrm{jrs}$ & $255 \mathrm{~cm}$ \\
Montréal & $21 / 11$ & $25 / 03$ & $122 \mathrm{jrs}$ & $235 \mathrm{~cm}$ \\
\hline
\end{tabular}

année concerne le Palais du Bonhomme, construit entièrement de glace. Le site a accueilli le nouveau concours international de sculpture sur glace. D'autres artistes ont exécuté dans la neige des oeuvres monumentales en démonstration à la Place de la Famille. Depuis quelques années, la majorité des activités extérieures se déroulent face au Parlement de Québec, à l'entrée est de Plainesd'Abrahamet dans leVieux-Québec.

\section{Le Vieux-Québec sous la neige... charme et plaisir}

En décembre demier a eu lieu le lancement d'unévénementhivernal prometteurintitulé: *Le Vieux-Québec sous la neige.... charme et plaisir». Cette activité, initiée par le Centre d'interprétation de la vie urbaine de Québec, vise à mettre en valeur la culture, les loisirs d'hiveret la féerie du Vieux-Québec pendant la période des fêtes. Les musées et centres d'interprétation, la Ville de Québec ainsi que certains partenaires privés participent au nouvelévénement. Lesprincipalesattractions mises de l'avant ont étế la crèche grandeur nature et l'immense sapin illuminé de la Place d'Armes, la glissade de la terrasse Dufferin, la patinoire de Place d'Youville, le rallye dans un jardin d' hiver, ainsi que l'exposition de crèches de Noell. Cetévénement, qui se veut annuel, s'est déroulé cette année du 15 décembre au 10 janvier.

\section{Le patinage}

La Ville de Québec a aménagé sur son territoire trente-cinq surfaces glacées dont trois méritent une attention particulière. Ce sont les patinoires de la terrasse Dufferin, de la Placed'Youville etdelarivière Saint-Charles.

Lors du réaménagement de la Place d'Youville en 1987, l' architecte paysagiste Georges Houplain a prévu un espace public qui, des les premiers jours de froid, deviendrait une patinoire de glace artificielle. La surface glacée dépasse de 65 mètres carrés la superficie de la célèbre patinoire extérieure du Rockfeller Center à New York.
En janvier, la rivière Saint-Charles se transformeen patinoire sur une distance de $1,7 \mathrm{~km}$ et traverse les quartiers populaires deQuébec. Cette année, elle a ouvert du 8 janvier à la fin de févnier. La candidature de Québec pour les Jeux de 2002 aurait motivé l'annonce de la réouverture de cette patinoire qui était fermée depuis quelques années en raison de restrictions budgétaires.

La patinoire de glace naturelle de la terrasse Dufferin, jadis très fréquentée par les personnalités qui logeaient au Château Frontenac, a été réouverte également dans le cadre des fêtes du Centenaire de cet hôtel de prestige.

Pour les plus frileux ou lors de mauvais temps, le centre commercial les Galeries de la Capitale a aménagé dans son parc d'amusements une vaste patinoire de glace artificielle ouverte au public sept jours par semaine. Les soirées avec grand orchestre attirent des centaines de patineurs d'âge mûr.

Enfin, la municipalite de Sainte-Foy, en banlieue de Québec, possède un anneau de glace artificielle de $400 \mathrm{~m}$ qui sert aux compétitions nationales et à l'entrainement des patineurs de vitesse. Ouverte de la mioctobre a la fin mars, elle accueille en soirée et durant les fins de semaine le grand public qui apprécie le fini de la surface glacée.

\section{Les glissades}

Depuis toujours, les Plaines d'Abraham ont permis àdes milliersd'enfants et souvent aux plus âgés de glisser sur les nombreuses pentes qui parsèment le célèbre parc des champs de bataille. Cette belle tradition a été renforcée par le Carnaval de Québec qui, dans les activités de la Place de la Famille, installe une grande glissade surnommée use-culotte.

A l'occasion du centenaire du Château Frontenac, la Villede Québec, conjointement avec le Service canadien des parcs, ont fait renaitre une autre vieille tradition en inaugurant au début de janvier, l'ancienne glissade réaménagée de la terrasse Dufferin. Cet 
equipement de bois et d'acier qui remonte au début du siècle avait été fermé en 1981. La glissade permet a trois traînes sauvages de dévaler simultanément dans le panorama grandiose entourant la terrasse.

\section{Le ski de randonnée}

Le territoire de la Communauté urbaine comple huit centres de ski de randonnée qui totalisent $332 \mathrm{~km}$ de pistes. Il est également possible de faire de la raquette dans la majorité des sites. Le centre de ski de fond de Charlesbourg offre à lui seul un réseau de $194 \mathrm{~km}$ de pistes a moins de dix minutes du centre-ville. En soiré, ilest possible de skier sur les Plaines d'Abraham et le long de la riviere Montmorency dans le camping municipal de Beauport.

En plein coeur de la cité de Champlain, le Parc historique des champs de bataille, communément appelé les Plainesd' Abraham, offre gratuitement quatre pistes familiales, deux pistes pour le patin et une piste de compétition. L’ensemble du réseau totalise $19,1 \mathrm{~km}$ de sentiers auquel s'ajoute un refuge chauffe avec salle de fartage. A la basseville, sur la rivière Saint-Charles, un sentier double est tracé en parallèle à la patinoire municipale.

\section{Le ski alpin}

Sous le theme aSkiez en ville et skiez souvent $\%$, la Ville de Sainte-Foy administre un centre familial de ski alpin situé à l'ouest de la côte Myrand. Les deux pentes d'une dénivellation de $30 \mathrm{~m}$ sont éclairées et équipées d'un télé-ski. Sur la rive Sud, à quelques kilomètres de la traverse de Lévis, la municipalite offre un centre identique situé sur la rue Mgr-Bourget.

\section{Les activités \\ hivernales en périphérie}

Les conditions climatiques extrêmes enregistrées dans les régions montagneusessituées à proximité de Québec favorisent la pratique d'activités traditionnelles telles le ski alpin, le ski de randonnée ou la raquette. Par ailleurs, après quelques années difficiles caractérisées par l'absence de réglementation, le sport de la motoneige connaît une popularité grandissante dans l'ensemble du Québec etattire de plusen plus de visiteurs européens à la recherche d'aventure.

\section{Le ski alpin et ses nouveautés}

La grande région de Québec compte maintenant quinze centres de ski alpin dont trois stations touristiques quatre saisons. Ce sont le Parc du Mont Sainte-Anne, la Station touristique de Stoneham et le Parc régional du Massif du Sud. Le développement récent d'un réseau de centres de ski alpin dans les Appalaches a fait disparaître le quasi monopole que détenaient les centres de la rive Nord. Le Massif de Petite-Riviêre-SaintFrançois avec sa dénivellation de $770 \mathrm{~m}$, ses onze pentes et ses deux nouvelles remontées mécaniques, pourrait très bientôt adhérer au rang des stations touristiques majeures.

LariveNordde Québeccompte actuellement sept centres de ski alpin, près de 130 pistes dont 65 éclairées en soirée. Le Parc du Mont Sainte-Anne conserve son titre de plus grand centre de ski alpin a l'est des Rocheuses avec sa nouvelle télécabine ultra-rapide et son télé-siège à bulle qui portent sa capacité horaire à 17760 skieurs. Cette station touristique a instauré un nouveau système de billetterie appelé Skidata. II permet aux skieurs d'acheter des points et de payer selon lenombre de remontéeseffectuées. Leskieur peut s'arrêter à tout moment et conserver pour une autre visite les points restants.

La Station touristique Stoneham, deuxième station à l'est des Rocheuses et classée cinquième au Canadapar lemagazine américain Snow Country, se distingue par laqualité de l'entretien de ses pistes et son festival de la neige qui souligne la venue du printemps. Stoneham sera I'hôte en 1993 de la coupe du monde de slalom.

Le centre de ski alpin Le Relais, voisin du célebreMont Saint-Castin, asubi récemment un développement intensif. De nouvelles remontées mécaniques ont été ajoutées et plusieurs pentes ont été ouvertes dont celles ducôttésud visibles du centre-ville de Québec.

La rive Sud de Québec compte six centres ou stations de ski alpin totalisant plus de 80 pentes. Le Mont Orignal, une des plus anciennes stations, s'est équipé récemment du premier télé-siè ge rapidesextuple au monde. LaGrande CoulEe, près de Montmagny, dont le sommet se situe à 853 mètres, est la seule station du réseau Appalaches à présenter des conditions de neige naturelle à $100 \%$. Le nouveau centre La Crapaudière se distingue en offrant de skier a l'heure.

Le nouveau parc régional du Massif du Sud, développé par la Corporation d'aménagementet de développement du Massif du Sud, offre sur son territoire de $103 \mathrm{~km}$ carrés, un centre de ski associe à de nombreuses activités de plein air dont l'equitation, les randonnées en carriole, la motoneige, la cabane à sucre et le ski de fond. La jeune station compte dix pistes au design très particulier et deux remontées mécaniques. Situé à moins de $90 \mathrm{~km}$ des ponts de Québec et PierreLaporte, le Massif du Sud est le centre de ski alpin le plus élevé du Québec avec ses 915 mètres d'altitude. La dénivellation de $400 \mathrm{~m}$ est cependant comparable a celle de Stoneham.

\section{La planche à neige}

Après quelques moments d'hesitation de la part des centres de ski alpin, le sport de la planche à neige ou surf des neiges, envahit graduellement les pistes de tous les centres de la région. LeMont Sainte-Anne, a reçu du 4 au 7 mars 1993 la Coupe du monde de surf des neiges sanctionnée par la Fédération internationale de surf des neiges.

\section{Le ski de randonnée et la raquette}

Herman Smith Johannsen, surnomme Jack Rabbit et initiateur du ski de fond au Canada, adéfriché en 1935 le premier sentier de ski de fond de la région de Québec à Lac Beauport. Aujourd'hui, notre région se distingue en Amérique du Nord avec ses $2000 \mathrm{~km}$ de sentiers répartis dans trente-deux centres. La pratique de laraquetteest possible également dans lamajoritédes centres mais onze offrent des sentiers balisés et entretenus à cette fin.

La rive Nord de Québec est le royaume des randonneurs avec vingt-deux centres de ski, 278 pistes totalisant $1636 \mathrm{~km}$. Le parc du Mont Sainte-Anne entretient un réseau de calibre intemational avec $235 \mathrm{~km}$ de sentiers réguliers et $136 \mathrm{~km}$ pour le pas de patin. Il était, en janvier, l' hôte de la Coupe du monde de ski de fond. Le Camp Mercier dans la réserve faunique des Laurentides possède $192 \mathrm{~km}$ de pistes et offre en plus trois sentiers de longue durée avec refuges en territoire sauvage. Les excursions propostes peuvent durer de deux a six jours.

Sur la rive Sud, le ski de randonnée est surtout pratiqué dans des centres de plein-air en association avec les stations de ski alpin. Les dix centres de la région offrent cinquante-cinq pistes totalisant $400 \mathrm{~km}$ de sentiers qui parcourent le paysage tranquille de terres agricoles ou celui plus tourmenté des Appalaches. 


\section{La motoneige}

Mise au point en 1959, les premiers engins appelés ski-dog, et plus tard ski-doo, contrastaient avec les sports traditionnels de la raquette et du ski. Aujourd'hui, des engins modernes, presque silencieux, circulent sur de véritables autoroutes de neige, entretenues par chacun des clubs reconnus par la Fédération des clubs de motoneige. La carte provinciale dessentiers provinciaux démontre l'ampleur du réseau Trans-Québec.

Biendesservie parce réseau, la grande région de Québec est sillonnée par différentes catégories de pistes totalisant plus de $4000 \mathrm{~km}$. Ces pistes sont parsemées de relais chauffés et reliés aux nombreux établissements hôteliers ou de restauration qui offrent differrents forfaits adaptés aux motoneigistes. De nombreuses entreprises dispensent des services de location avec guide pour les excursions hors pistes surtout appréciées des clientèles europeennes. Des villages d'accueil tels Saint-Eugène de l'Tslet et Saint-Ubalde dans Portneuf reçoivent des motoneigistes qui désirent séjoumer dans les familles.

Sur la rive Nord, l'ensemble du réseau atteint $1500 \mathrm{~km}$. Les pistes provinciales numéro 3 , 13 et 23 relient la région de Québec à Charlevoix, au Saguenay-Lac Saint-Jeanet à la Mauricie. Situé a la jonction des pistes provinciales en direction nord et ouest, le territoire de Portneuf est lesite parexcellence de la motoneige avec son réseau de $1000 \mathrm{~km}$ et l'importante infrastructure d'accueil mise en place. La carte de 1993, publiće par la Régionale des clubs du comté de Portneuf, diffuse toutes les informations relatives a la pratique de cette activité.

Larégion touristique Chaudière-Appalaches, véritable paradis de la motoneige, domine avec ses $2500 \mathrm{~km}$ de pistes. Les sentiers provinciaux dont les numéros $5,25,55,65$ et 75 parcourent le paysage agricole de laplaine du Saint-Laurent, suivent les vallées ou s'enfoncent à travers le massif des Appalaches. Deux grands circuits thématiques sont offerts aux fervents de la motoneige soit le Sentier des érablières et le Sentier de Beauce-Appalaches d'une longueur de plus de $550 \mathrm{~km}$ chacun. La carte de la 7e édition (1993) contient de précieux renseignements sur les sentiers, les relais et les établissements commerciaux offrant des services.

Le festival nord-américain de la motoneige s'est déroulé à Thetford-Mines du 27 au 31 janvier 1993 tandis que la région de Saint-
Georges en Beauce a reçu le Championnat d'accélération Canada-Etats-Unis les 6 et 7 février 1993.

\section{La carriole ou le traîneau}

Plus traditionnelles, les activités des centres d'équitation se poursuivent souvent en hiver en offrant les sleigh ride. Ces ballades en carriole ou en traineau sont offertes à de nombreux endroits tels a Lac Beauport, à Stoneham, à Grondine et au ranch Massif du Sud. Tous ces centres sont identifiés dans les guides touristiques sous l'item équitation.

\section{Les glissades sur neige}

Une activité pratiquée par tous les jeunes, la glissade sur chambre à air, sur carpette ou en traîne, se pratique de plus en plus dans la régiondeQuébec. Lesremontéesmécaniques offertes, surtout dans les centres ou bases de plein-air, permettent aux adeptes de tous les âges de retrouver les joies de l'enfance.

Le Village des Sports de Valcartier situé à 20 minutes de Québec, est le plus grand centre de glissade au Canada avec ses 21 pentes de neige dont l'Everest qui serait la plus haute glissade en Amérique du Nord. Cette entreprise privée a aménagé également une patinoire de $1,5 \mathrm{~km}$, une piste de course sur glace pour les mini-formules motorisées et $40 \mathrm{~km}$ de sentiers de ski de randonnée.

La région compte d'autres sites de glissade surneige, avec remontées, dont ceux du Club Mont-Tourbillon à Lac Beauport, du Manoir duLacDelage, duCentre nature Saint-Basilede-Portneuf, des glissades de Lotbinière et du Mont Orignal. A Tewkesbury, les Excursions Jacques-Cartier organisent du rafting sur neige. Il s'agit d'une descente rapide sur une pentedeneigedans uncanot pneumatique pouvant accueillir de huità douze personnes. La remontée s'effectue en carriole.

\section{Interprétation et safaris}

Les centres éducatifs, les bases de plein-air et certaines entreprises privées offrent des activités d'interprétation de la nature ou des safaris d'observation de la faune. L'entreprise axpédition Québec-Faune de Beauport organise, notamment pour les touristes européens, des expéditions qui permettent de vivre au rythme de la nature et d'observer la faune dans le parc de conservation de la Jacques-Cartier. En compagnie de naturalistes Equipés d'instruments modernes, il est possible de retrouver la piste des loups et d'admirer l'orignal ou le lynx dans leur habitat naturel.

Sur larive Sud, Aventure Nord-Bec de SaintLambert de Lévis offre des forfaits comprenant l'initiation à la conduite d'attelages de chiens. Des expéditions deplus longue durée sont offertes avec camping d'hiver sous la tente.

\section{La pêche blanche}

La saison de la pêche d'hiver dans la région de Québec a débuté cette année le 20 décembre. Les plans d'eau accessibles sont le fleuve Saint-Laurent et une dizaine de lacs situés sur les rives Sud et Nord. Il est Également possible de pêcher la truite d'élevage à l'année longue dans les nombreux étangs aménagés de la région de Québec.

\section{Conclusion}

En somme, Québec peut à juste titre mériter l'appellation Capitale de la neige. En effet, il y existe une quantité impressionnante d'activitéshivernales, d'attractionsetde services, notamment dans les régions périphériques de Québec.

Malgré les rigueurs de l'hiver, les Québécois ont développé au cours des années, des infrastructures de plein air et des activités originales qui ont favorisé la participation populaire et attiré l'attention de nombreux visiteurs. Après les raquetteurs et le ski alpin, le Carnaval de Québec a tracé la voie à de nombreux entrepreneurs hardis quin'ont pas craint de relever le defi d'un produit hivemal de qualité. Les nouveaux vêtements d'hiver, chauds et légers, ont fait disparaître les demières craintes exprimées par les amateurs plus réticents. Aujourd'hui, des milliers de sportifs dévalent les pentes, sillonnent les sentiers ou glissent sur lessurfaces glacósesdespatinoiresoudesrivières.

Enfin, incitées par les besoins nouveaux exprimés par les clientèles intemationales, des entreprisesdelarégionont développédenouvelles initiatives. Citons en particulier les grands périples hors des sentiers battus, le camping d'hiveretles randonnées en traineauàchiens ou l'escalade de falaises de glace. La motoneige, apres la crise des annees 1980, attire de plus en plus d'adeptes et permet à beaucoup d'établissements hôteliers d'afficher un taux d'occupation relativementélevé. Québec, Capitale de la neige, voilà un titre mérité pour une région qui offre desomais un produit quatre saisons. Pour un grand nombre d' intervenants touristiques, la saisonmorteçapourraitneplusexisteràQuébec. 